\title{
Enhancing Discovered Process Models with Data Object Lifecycles
}

\author{
Dorina Bano*, Francesca Zerbato ${ }^{\dagger}$, Barbara Weber $^{\dagger}$ and Mathias Weske* \\ ${ }^{*}$ Hasso Plattner Institute, University of Potsdam, Potsdam, Germany \\ Email: dorina.bano@hpi.de, mathias.weske@hpi.de \\ ${ }^{\dagger}$ University of St. Gallen, St. Gallen, Switzerland \\ Email: francesca.zerbato@unisg.ch, barbara.weber@unisg.ch
}

\begin{abstract}
Process discovery is an important area in the field of process mining, where most discovery algorithms focus on process control-flow, giving little attention to the data-flow perspective. As a result, the discovered process models lack information about data dependencies, and process experts need to manually enrich the discovered process models accordingly. This requires deep domain knowledge, is not scalable, and error-prone. To overcome this limitation, this paper proposes an approach that aims to discover the data objects and their behavior by investigating how event attributes are manipulated during process execution. The resulting data objects are used to enhance the discovered process model. The feasibility of the proposed approach is evaluated with two real-life event logs: Road Traffic Fine Management and Hospital Billing.
\end{abstract}

Index Terms-process mining, business process, data object, data object lifecycle

\section{INTRODUCTION}

Process discovery is an important part of process mining, deriving knowledge about business processes from execution data stored in different information systems. Usually, this data is extracted from data stores and represented as event $\log s$, which contain sequences of events capturing the execution of business process instances (i.e., cases).

Events in an event log are associated with attributes, some of which can be mandatory [1], for instance (1) the case identifier, which correlates several events into a single case, i.e., a process instance; (2) the activity name, which identifies a welldefined execution step of the process; and (3) the timestamp, which indicates when the event occurred [2]. Depending on the process mining goal, events can be associated with additional attributes, such as the resource, describing the person or device responsible for executing a specific activity, or the department, defining where the activity has been executed.

Event logs are used as an input for different types of process mining, i.e., process discovery, conformance checking, and enhancement [3].

Process discovery allows to discover a process model from an event $\log$ [4]-[6]. However, conventional process discovery algorithms tend to focus on the control-flow, giving less attention to the data-flow perspective [7], i.e., the data associated with events and their dependencies. As a result, the discovered process models lack information about which data is read or written by process activities or how many process instances require a certain data object to be executed. Process experts need to manually enrich the models with such dependencies, but this requires deep domain knowledge, is not scalable, and can be error-prone.

Still, the data-flow is crucial to understand the actual execution of processes and, in consequence, the discovered process model. Indeed, a holistic view combining controlflow and data-flow can support process experts in making decisions [8], e.g., related to the modeling, logging, and extraction of process execution data [9], or in reasoning about data dependencies [10], [11] and their consistency [12]-[14]. Besides, we argue that knowing which activities modify which data objects can help analysts with post-discovery analyses, e.g., to focus on specific paths, find root causes for deviations, or split the event logs into sub-logs.

Research on data-aware process discovery has focused on approaches to discover the data-flow perspective from event logs, e.g., to identify rules that explain why a certain process path is executed [7], [15] or to discover infrequent paths [16]. However, these approaches mainly focus on deriving databased conditions that affect the control-flow rather than representing the data-flow on the final process model.

In this paper, we propose an approach that leverages existing process discovery algorithms to discover not only the process model but also the data objects and their lifecycles by considering an event $\log$ as a unique source of information. To achieve this, the paper analyzes how the attribute values change within each case, reflecting how the business process activities modify data. We argue that the business process can be understood much better when the process expert is equipped with the discovered process model and the data objects lifecycles attached to this process.

The remainder of this paper is organized as follows. In Section II, we explain the preliminary notions needed to understand the rest of the paper. Section III describes a running example used to introduce the proposed approach. In Section IV, we detail the steps of our approach to discover the data objects and their lifecycles from an event log. Then, in Section V, we illustrate the application of our approach to two real-life event logs. Related work is discussed in Section VI, while Section VII concludes the paper. 


\section{PReliminaries}

In this paper, we focus on discovering data objects and their lifecycles from an event log. Here we recall some basic notions that are relevant to follow our approach.

The main input artifact of any process mining technique is an event log. An event log is a collection of cases, each representing the execution of a business process instance. Each case consists of a sequence of events, where each event captures the execution of a specific business activity within the case. The standard format for storing, sharing, and analyzing event $\operatorname{logs}$ is the eXtensible Event Stream) (XES) [1]. The attribute values represent the relations between events and attributes in an event log. Within the same case, attributes can be associated with single events or refer to the case as a whole. The former ones are called event attributes and their value can vary based on the process step (event) being executed. The latter ones are called case attributes and are invariant, i.e., they do not change as the events of the case occur [3].

Besides event $\log$, another typical artifact of process mining techniques is process models, which can be either obtained from event $\log$ s as a result of process discovery or used as an input for conformance checking and enhancement. Process models describe the activities aimed to achieve a particular business goal and the execution constraints among them, i.e., the control-flow, using different notations, such as the Business Process Model and Notation (BPMN) [17], [18].

Aside from the control-flow, process models can represent other aspects, such as the data-flow, i.e., the data modified by process activities and exchanged between them. In BPMN, the data created and used by process activities is represented through data objects. They are connected with activities via directed data associations, represented as edges. Based on the edge direction, read/write accesses are defined. An edge from a data object to the process model activity represents a read access to the data object. Alternatively, an edge from an activity to the data object represents a write access. Write access defines the creation of a new data object or the modification of an existing one [11]. Data objects in a process can be annotated with their state, which can change at different points during process execution. To be executed, a process activity might require that a specific data object is available in a particular state. Once the activity is completed and has consumed the data object, the data object state might be updated. The combination of different data object states and the possible transitions between them is usually referred to as the data object lifecycle.

The approach to discover the data objects and their lifecycles by considering only an event $\log$ as a single source of information is described in Section IV.

\section{RUNNING EXAMPLE}

In this section, we introduce a running example inspired by a real-world healthcare process, which we will use to motivate and explain the approach proposed in Section IV.

In the top part of Figure 1, we show an excerpt of an event $\log$ capturing the execution of a Patient Management (PM) process for patients with suspected appendicitis. The case ID attribute groups all the events related to one case, e.g., "001", and it is a mandatory attribute, together with activity and timestamp. Attributes such as gender, age and pregnancy are case attributes, as we assume that they are invariant within a case. Instead, attributes such as risk, and bodyTemp are event attributes, as they refer to single events and, thus, can change value multiple times within a case.

The lower part of Figure 1 shows a simplified PM process model (represented as BPMN), which depicts the main steps of the process. For the purpose of this example, we list the data attributes modified by the process activities as text annotations. The diagnosis of appendicitis is mainly clinical and begins with an analysis of signs and symptoms collected by the physician during a Physical Examination (A). Examples of collected information are the patient's age and gender, the pregnancy status in case the patient is a female, and other signs indicative of appendicitis, such as presence of fever. Based on the findings of the physical examination, the physician determines the risk using a clinical scoring system and decides if an antibiotic prophylaxis is needed. If the physician determines that the risk is high, the patient must immediately undergo Urgent Appendectomy (B). Otherwise, further laboratory and imaging diagnostics are conducted to rule out other conditions. An Imaging Exam (C), chosen among ultrasounds, computed tomography (CT), or magnetic resonance imaging (MRI), is carried out to help confirm appendicitis. For pregnant patients, MRI is always preferred over CT. At the same time, Blood Analysis (D) is conducted to check for values indicating infection, such as C-reactive protein (CRP) levels or high white blood-cell count (WBC). Then, based on the results of the blood analysis and the imaging diagnostics, the physician needs to Make a Diagnosis (E). In case of uncomplicated appendicitis, the patient can be treated with an Antibiotic-first Approach (F) if not pregnant. Otherwise, a Regular Appendectomy (G) is performed under anesthesia, keeping track of whether the appendix was perforated or not. After appendectomy or antibiotic-first treatment, a Clinical Assessment $(\mathrm{H})$ is performed to re-evaluate the patient, give indications for home-care, and, in some cases, prescribe post-operative antibiotics.

During execution, the process creates and modifies data attributes. For example, the event attribute bodyTemp is modified by activities (A), (B), (G), and (H). In the PM process model, the body temperature would be part of a data object (represented in red in Figure 1), connected to the activities mentioned above through data associations and showing a particular state based on the process activity modifying it. However, on the one hand, by solely looking at an event log, it is not easy to understand how data attributes can be represented and grouped into data objects and how these data objects evolve as a result of activity execution. On the other hand, by looking at the process model, it is not trivial to understand how many process instances require access to a particular data object, e.g., how many cases write the pregnancy attribute. Still, the information provided by an event log can be valuable 


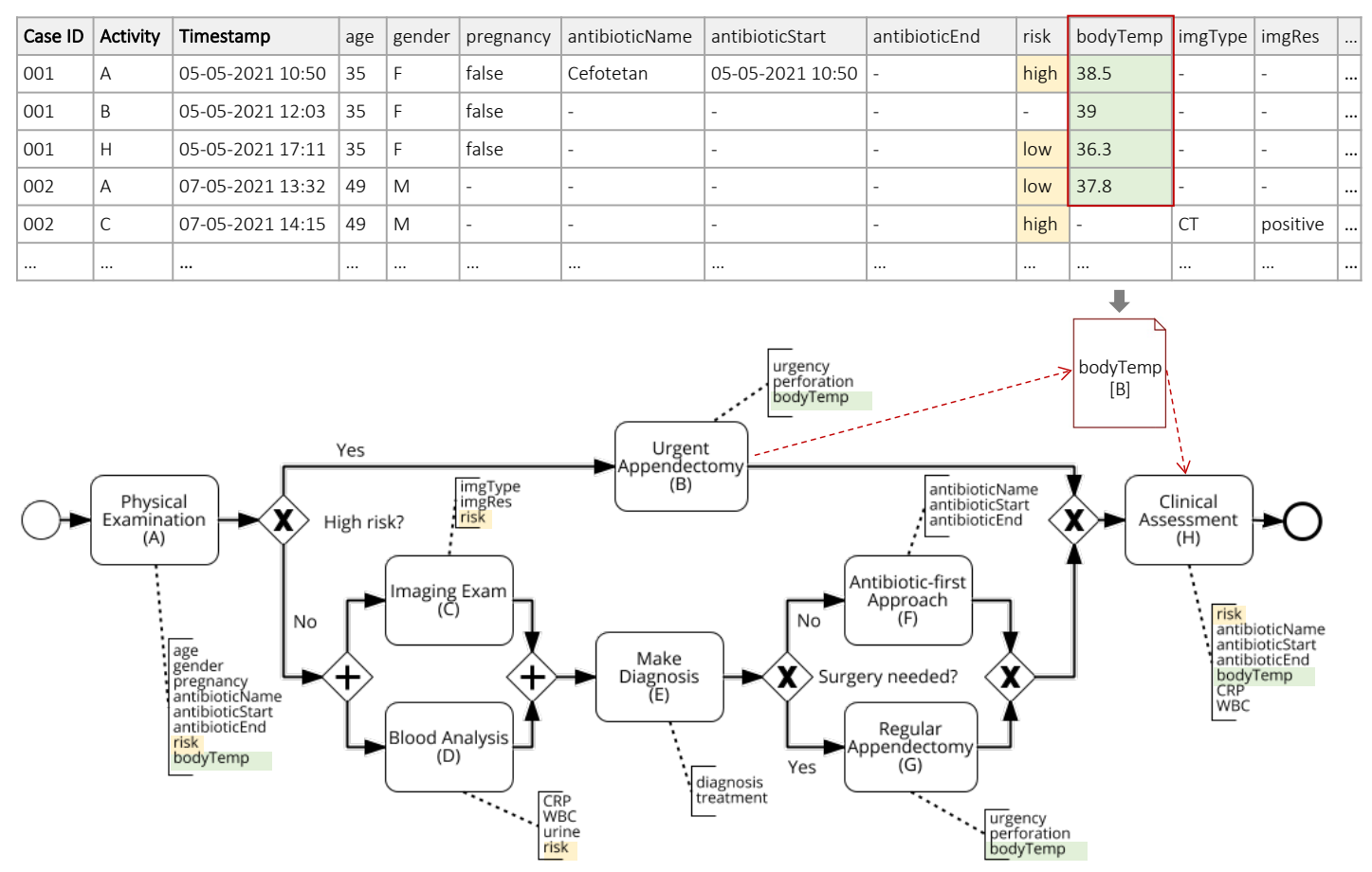

Figure 1: (Top) Example of event log excerpt related to the Patient Management (PM) process with suspected appendicitis. Case ID, Activity and Timestamp are mandatory attributes in this log. (Bottom) Simplified PM process related to the event log.

to group attributes into the data objects and to identify optional data attributes. In the next section, we introduce an approach that aims to close this gap by discovering the data objects and their lifecycles directly from an event log. The discovered data objects are used to enhance a discovered process model and complement it with statistics about how many process instances require access to a particular data object.

\section{DisCOVERING DATA OBJECTS AND THEIR LIFECYCLES FROM AN EVENT LOG}

The discovered process model (e.g., PM process explained in Section III) does not contain explicit information on how the activities consume the data during the process execution. Therefore, in this section, we show how this information can be captured from an event log and later used to enhance the process model, which is upfront discovered from the same event log. We track how the event log attribute values change within the case. Based on the discovered attribute behavior and a set of steps, we propose an approach to group the event $\log$ attributes into data objects and, afterwards, their lifecycle is derived. Our approach is described in the following steps, which are sketched in Figure 2.

a) Attribute-access event log extraction: The starting point of our approach is an event log. The association between events and attributes in the event log is explicitly defined by the attribute values. Within the same case an attribute value can be invariant or modified by another upcoming event. To understand the behavior of data attributes within an event log,

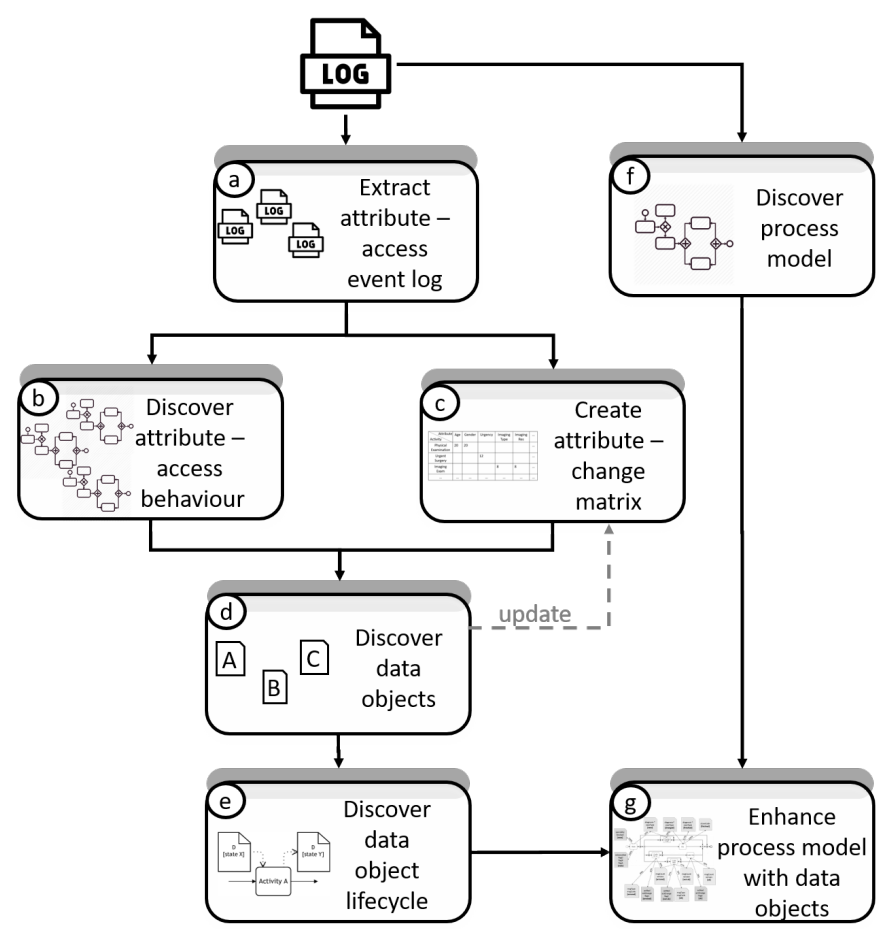

Figure 2: Overview of the proposed approach.

we first split the log into event log chunks, each pertaining to a different event $\log$ attribute. Activities (i.e., events) that do not write or modify the value of any optional attribute are left 
out as they do not play a role in determining the behavior of the attributes. All the other activities, together with the case identifier, timestamp and the corresponding attribute values, are stored in a new event $\log$, called Attribute-Access Event $L o g$, hereinafter referred to as $A A E L$. This implies that the number of generated AAELs will be the same as the number of optional attributes available in the original event log.

b) Discovering attribute-access behavior: After having obtained an AAEL for each attribute in the original event log, we apply traditional process mining algorithms, such as the Inductive Visual Miner [5], to discover the attribute-access behavior. Our language of choice to represent the discovered behavior is BPMN [17], [18] as it allows us to discover the complex behavior (i.e., exclusive and parallel gateways) - a feature that will turn useful when representing data objects in the process model (cf. Step g). A design alternative would be to use the Directly-Follows Graph (DFG) (since it captures how many events or cases have write access to a specific attribute) instead of BPMN, but our goal is to capture the generalized behavior of the data attributes, including exclusive and parallel behavior.

If the discovered process models contain activities with very low frequency (i.e., activities that are executed for a low number of cases or events) compared with other activities a threshold might be necessary for filtering out those activities.

c) Attribute-change matrix creation: The BPMN process models discovered in the previous step capture the attributeaccess behavior showing how process activities are writing or modifying a specific event log attribute (i.e., (over)write a particular attribute). However, they do not provide any information regarding the number of cases in which the attribute is accessed by a certain activity. Indeed, considering only the attribute-access behavior to group the event log attributes into data objects is necessary but not sufficient as two attributes might have the same access behavior, but the number of discovered cases for each attribute can be different. To additionally capture this, we construct an attribute-change matrix. This matrix is used together with the attribute-access behavior to group the event log attributes into data objects (see Figure 2).

The attribute-change matrix depicts the number of times a certain activity accesses (i.e., writes, modifies) an attribute in

\begin{tabular}{|c|c|c|c|c|c|c|c|c|c|}
\hline Activity & 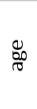 & 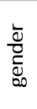 & $\frac{\substack{\frac{2}{2} \\
\frac{0}{2}}}{0}$ & $\sum_{\substack{\infty \\
\underline{\underline{m}}}}^{\infty}$ & 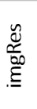 & 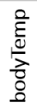 & 慈 & 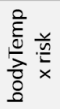 & $\ldots$ \\
\hline Physical Examination (A) & 20 & 20 & 20 & & & & & & $\ldots$ \\
\hline Urgent Appendectomy (B) & & & 15 & & & & & & $\ldots$ \\
\hline Imaging Exam (C) & & & & 8 & 8 & 12 & 12 & $x$ & $\ldots$ \\
\hline Body Analysis (D) & & & & & & 19 & 19 & $x$ & \\
\hline$\ldots$ & $\ldots$ & $\ldots$ & $\ldots$ & $\ldots$ & $\ldots$ & & & $\ldots$ & $\ldots$ \\
\hline
\end{tabular}

Figure 3: An example of attribute-change matrix based on the running example. The numbers in the matrix indicate how many times each activity (over)writes on a specific attribute. each AAEL. The matrix is structured as a table where rows represent all activities and columns represent all attributes. The number of columns in the matrix is the same as the number of AAELs. Each row in the matrix is populated with the frequencies that represent how often the attribute is accessed by the respective activities.

An example of attribute-change matrix is illustrated in Figure 3. The rows represent some of the activities taken from the running example presented in Section III, while the columns represent some of the additional attributes. As shown in Figure 3, the Physical Examination (A) activity is (over)writing the value of the age attribute 20 times in the AAEL. The same activity, for the same number of times is (over)writing the gender attribute. This is an indication that these two attributes might belong to the same data object. However, without looking at their individual behavior we cannot guarantee that this is true. This is why the matrix complements the behavior with the ultimate goal of grouping the attributes into data objects.

d) Data object discovery: This step aims to aggregate the event log attributes into the data objects by considering the attributes-access behavior and attribute-change matrix obtained in the previous steps (see Figure 2). The discovery of data objects starts by taking into consideration the case attributes. To identify them in the matrix, we search for all attributes that are written only once per case. It might happen that the attribute value is overwritten by the same activity within the same case and this information is not explicitly captured by the attribute-change matrix. Therefore, the attribute-access behavior has to be taken into account and checked if the discovered process model activity contains a self-loop. If this is the case, the attribute is not considered as a case attribute because within a case its value is overwritten by the same activity name.

For the identified case attributes, we consider grouping them in one data object if the frequency of the activities that are accessing these attributes is the same. In some cases, it might happen that the frequency is not exactly the same but varies by a few units. For example, one activity is accessing one attribute with frequency 2743 and another attribute with frequency 2741 . This might happen due to incomplete cases being extracted in the original event log (e.g., if some cases were still open when the database was dumped [19]) or can be the result of data quality issues [20]. Therefore, whenever we compare the activity frequencies, we suggest considering a reasonable threshold, which can be set by the process expert.

As the last step, we check the event timestamps to ensure that the attribute values are written roughly simultaneously within one process instance. If this is true, then the case attributes under consideration are assigned to the same data object.

For example, if we consider the attribute-change matrix illustrated in Figure 3, we can see that the case attributes age and gender are both accessed by the same activity (i.e., Physical Examination (A)). If we are sure that the attribute 


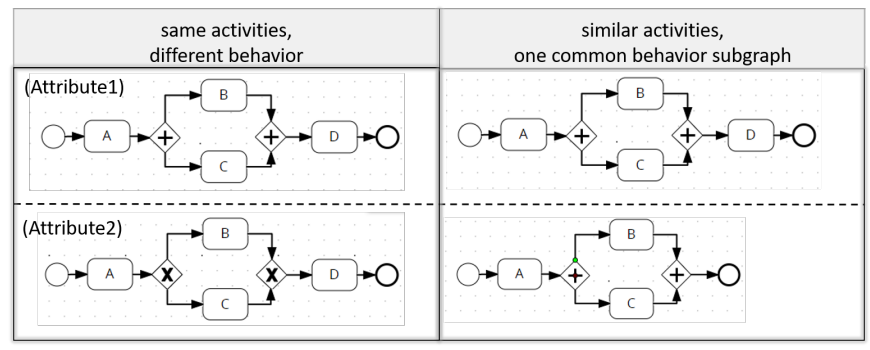

Figure 4: Two cases of discovered attribute-access behavior

access behavior is the same and after checking timestamps we see that they are also accessed simultaneously by the same activity, then we can group them in one data object.

Once all case attributes are grouped into the respective data objects, the next step is to look at the event attributes. Different from case attributes, event attributes are accessed by more than one activity or multiple times by the same activity (within the same case). Therefore, besides the absolute frequency the order under which these attributes are modified is essential for determining the data objects. To capture such information, we compare the discovered attribute-access behaviors with each other and distinguish the following two cases.

- Same activities, same behavior.

From the attribute-change matrix, we can group the attributes that are accessed by exactly the same activities with the same frequency. Afterwards, their access behavior is compared to determine their similarity in terms of activities and control-flow. Based on the similarity result, the attribute-change matrix is updated to store such information as follows: an additional column that represents the group of attributes with similar behavior is added. Considering the matrix illustrated in Figure 3 (gray column), we can understand that the bodyTemp and risk attributes have similar behavior. Therefore, the total number of added columns is equal to the number of attribute groups with similar behavior.

As the last step, for each group we check if the same activities are accessing the group attributes simultaneously (i.e., within the same case). If that holds, then most likely, these attributes will belong to the same data object. Otherwise, the attribute groups will be further divided until the above holds. Finally, each group defines one data object, even when the group size is equal to one (data object is created as a result of one event attribute). An example of two attributes-access behaviors which involve the same activities but different behavior is illustrated in the first column of Figure 4. The discovered behavior for both attributes contains the same activities (i.e., named $A, B, C, D)$ but different behavior. For the first attribute, A occurs, and after that, a parallel gateway is discovered, which implies that $B$ and $C$ will be executed, and the process will end with the execution of activity $D$. In this example Attribute 1 and Attribute 2 are assigned to different data objects. If the attribute- access behavior for these two attributes would have been the same (i.e., they would have both included an exclusive or parallel gateway), then these two attributes would have most likely belonged to the same data object.

- Similar activities, one common behavior subgraph.

In this step, we look for attributes that are accessed by a similar set of activities, that is, they share some common activities but do not have exactly the same attribute-access behavior. For example, the first attribute is accessed by five activities and the second attribute is accessed by three of those five activities and three additional ones that are not accessing the first attribute. This implies that the behavior of these two attributes cannot be identical.

However, we can still look for a common sub-behavior and argue whether these attributes can be part of the same data object. For that, it is important to check if we can identify a common subgraph between these two attributeaccess behaviors. More specifically, we are looking for a subgraph that contains at least two activities and at the most the same number of activities of the smallest behavior. For simple graphs representing attribute-access behavior, the comparison can be made visually. However, if the graphs are complex, approaches based on causal footprints [21] or refined process structure trees [22] may be used.

Once the subgraph is computed, the matrix is updated to capture such a group of attributes to ensure that the subgraph also represents the concurrent access of these attributes in the event logs. This means that all activities of the subgraph are accessing the attributes simultaneously within the same case. The process expert, who is equipped with the common subgraph and the matrix, can decide whether to create a new data object from these groups of attributes. If so, the attribute that is not always accessed from an activity of the common subgraph is marked with * sign in the corresponding data object. That is because these attributes are not always accessed every time the data object is accessed. Otherwise, the process expert can decide not to put these attributes as part of the same data object.

Since the data object might be a composition of several event/case attributes, coming up with a representative data object name is left to the process expert. However, an NLP approach can be used to support the process expert, for example the one in [23]. In the simplest case, the data object with a single attribute can share the same name with the attribute.

Figure 4 depicts an example of two attribute-access behavior which have similar activities and share one common subgraph (second column in Figure 4). Activities $A, B$ and $C$ are discovered in both attributeaccess behaviors. First the activity $A$ occurs. Afterwards, a parallel gateway is discovered, which implies that $B$ and $C$ will be executed. For the first attribute, the behavior has another activity called $D$. It is important to point out that 
both the discovered behaviors share a common subgraph. Since activities $A, B$ and $C$ are accessing Attributel and Attribute 2 simultaneously within the same case, then these attributes can be assigned to one data object. Since Attributel is not always accessed from an activity of the common subgraph then is marked with * sign in the corresponding data object.

e) Data objects lifecycle discovery: Once the data objects are discovered, their lifecycles are identified. The data object lifecycle captures the data object states and the state changes, representing how the data object is being transformed during process execution. We rely on the attribute-access behavior of each attribute involved in a single data object to derive its lifecycles.

The data object state constitutes the collective values of its composing attributes after each activity that manipulates any of these values. Specifically, for each activity discovered in the attribute-access behavior, a new data object state is created and named after the activity label. This implies that if the data object is created as a result of grouping only case attributes, then its lifecycle consists of a single state.

Otherwise, when the data object is defined as a set of event attributes, excluding the event attributes modified by the same activity within the same case, then any data object lifecycle has at least two states. For each activity involved in the attributeaccess behavior, a new state is created. For the data objects that involve optional attributes, the number of states is the same as the number of activities of the biggest (i.e., in terms of the number of activities) attribute-access behavior.

The name of the data object state is based on the activity accessing the data objects (i.e., discovered in the attributeaccess behavior). If the activity name follows an actionbusiness object pattern [24], we recommend using the past tense form of the action as a data object state name.

f) Process model discovery: In this paper we are aiming to discover the data objects and their lifecycle from an event $\log$. To represent the discovered data objects from the previous explained steps a process model from the original event log has to be discovered first. Similar to the second step of our approach (cf. Step b) we use any traditional process mining technique to discover the process model.

g) Enhance process model with data objects: Once the data objects are created, the next step is to represent them in the process model that is previously discovered from the original event $\log$ (cf. Step f). To achieve this, the data object lifecycle is used to map the data objects into the process model. Two cases are considered and explained in more detail below.

- Mapping the data objects derived from case attributes. To establish the connection between the activity that accesses the data object and the data object itself, a data output edge is used with the activity as its source and the data object as its target. This is because the data object is only written once by this activity and never updated for the rest of the process execution.
- Mapping the data objects derived from event attributes. To map these type of data objects to the process model we distinguish three behavioral patterns:

- Sequential access: If the data object lifecycle manifests a sequential behavioral pattern between pairwise two states (i.e. one state follows another state but not vice versa), then the data object is output from the first respective activity (the activity from which the state is derived) and it is read from the second respective activity before it is output by it in the new state. That is because the second activity has to read the state of the data object before updating it. For the read access, the data input edge is used in the model, which has the data object as a source and the second activity as a target.

- Exclusive access: If the data object lifecycle manifests an exclusive behavioral pattern between pairwise two states (i.e., if a state is reached, then the other state is never reached and vice-versa), then each activity outputs the data object with the corresponding state. The activity does not read the state of the data object that is output from each of them. It may happen that these activities are manifested in a different behavioral pattern in the process model, e.g., in parallel. It is exactly in this case where our approach helps the process experts to understand the behavior of the process beyond what is originally captured in the process model and perhaps even update the process model in order to be consistent with the data-flow. See the discussion in Section V on why this may happen.

- Parallel access: If the data object lifecycle manifests a parallel behavioral pattern between pairwise two states (i.e., one state is reached from another state and vice versa), then we distinguish two cases:

* Parallel activities: If two activities are in parallel in the process model, then this is not desirable because whichever activity updates the data object last overwrites the previous state that is output by the other activity. Therefore, the first state of the data object is permanently lost. This is an antipattern, which is desirable to be avoided.

* Exclusive to sequential activities: If, however, there is an exclusive gateway in the process model that leads to two different paths, where one includes a sequence of the activities and the other one contains the opposite sequence, then each path is considered as a sequential behavior pattern, and it is treated like shown above (i.e., parallel activities). For example, a decision is made upfront based on the customer to a) retrieve the payment then send the shipment or b) send the shipment then retrieve the payment.

- Self loops: If the discovered attribute-access behavior contains self-loops associated with activities, then, 


\begin{tabular}{|c|c|c|c|c|c|c|c|c|c|c|c|c|c|c|c|c|c|c|}
\hline 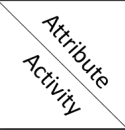 & 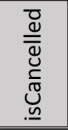 & $\begin{array}{l}\frac{n}{4} \\
0 \\
\frac{0}{00} \\
\frac{\pi}{0}\end{array}$ & 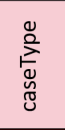 & 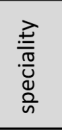 & $\begin{array}{l}\frac{\bar{d}}{\tilde{d}} \\
\frac{0}{0}\end{array}$ & 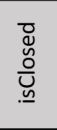 & $\begin{array}{l}\text { b0 } \\
\underset{\varpi}{\leftarrow}\end{array}$ & $\begin{array}{l}\frac{\infty}{00} \\
\underset{\pi}{\leftarrow}\end{array}$ & $\begin{array}{l}\frac{\widehat{6}}{60} \\
\frac{\mathbb{\pi}}{4}\end{array}$ & 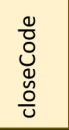 & 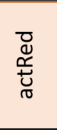 & 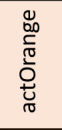 & 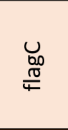 & $\begin{array}{l}\vec{c} \\
\overline{0} \\
\text { 品 } \\
\text { है }\end{array}$ & 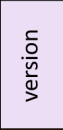 & 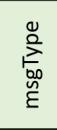 & 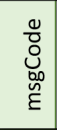 & 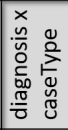 \\
\hline New & 77627 & 35528 & 77664 & 77627 & 77627 & 77661 & 77627 & 77627 & 77627 & & & & & & & & & $\mathrm{x}$ \\
\hline Fin & & 341 & 71 & & & & & & & 71619 & & & & & & & & $\mathrm{x}$ \\
\hline Change Diagn & & 45451 & 1467 & & & & & & & & & & & & & & & $x$ \\
\hline Code ERROR & & & & & & & & & & & 60 & 60 & 60 & 60 & 62 & 60 & 60 & \\
\hline Code OK & & & & & & & & & & & 63169 & 63124 & 63208 & 63746 & 64867 & & & \\
\hline Code NOK & & & & & & & & & & & 2992 & 2988 & 2994 & 3058 & 3330 & 1546 & 1548 & \\
\hline
\end{tabular}

Figure 5: Attribute-change matrix created for the Hospital Billing event log (color figure is available online).

from a data-flow perspective, they can represent two different situations:

* The activity is executed multiple times, modifying the data attribute at every execution. This corresponds to a loop in the control-flow of the original process.

* The activity is executed once but modifies several instances of the same data attribute, e.g., writing different values in batch. This corresponds to several rows in the original event $\log$ that refer to the same activity, have consequent yet close timestamps and write different values. To represent this behavior in the original discovered process model, a multi-instance data object can be used.

Holistic view generation. In the discovered process model from the original event log, besides discovering the data objects and their lifecycle, we are aiming to provide some statistics. More specifically, for each data object state, we capture the access ratio in percentage - the number of times a business process activity accesses the output data object over the total number of times that this activity occurs in the original event log. If the data object contains an optional attribute, then this information is provided for the attribute with the highest frequency. This information can be taken from the attribute-change matrix. The access percentage is appended to the data output edge connecting the activity with the data object. This information is helpful for the process expert to understand the involvement of activities in the data object manipulation during business process execution and the importance of the data in the overall process execution.

\section{Evaluation}

To evaluate our approach in terms of feasibility we use two real-life event logs, namely the Hospital Billing event log [25] and the Road Traffic Fine Management event log (RTFM) [26].

The Hospital Billing event log contains events regarding the billing of hospital services and includes 100.000 cases (451.359 events) that are processed over a three-year time period. Following the first step of our approach, the attributeaccess event logs (AAELs) are generated for all optional attributes except resource and activity lifecycle attributes because they describe respectively who executes the activity and the activity states. This step is implemented in the PM4Py framework [27] and is available on the GitHub ${ }^{1}$ repository. In total there are 17 AAELs extracted, which overall involve 6 activities out of the 18 happening in the original event log.

For each AAEL the Inductive Visual Miner [5] algorithm is applied and the attribute-access behavior is discovered. For understandability reasons, the activities that do not access any attribute are removed from the process model without affecting its behavior. Following the next step of our approach (cf. Step c), the attribute-change matrix is created and depicted in Figure 5.

Then, the data object discovery step takes place (see Figure 6). There are six data objects discovered in total. One data object is created to group together case attributes and the rest are created based on the event attributes. There is only one data object which contains a single optional attribute. Specifically, diagnoses and caseType attributes are assigned to the same data object (see Figure 6) where the diagnoses attribute is marked as optional because it is not accessed the same number of time as the caseType attribute. In addition, there is a common subgraph between the discovered attribute-access behavior of these attributes and the discovered process activities are accessing these two attributes subsequently. As shown in Figure 6, there is also a common subgraph discovered between the data objects containing the msgType, msgCode attributes and the data objects created as a result of actRed, actOrange, flagC attribute but in the common subgraphs activities are not accessing simultaneously these attributes. Therefore, they are stored in two different data objects.

Subsequently, the process model is discovered from the original event $\log$, which is illustrated in Figure 7 . The discovered process model reveals that many of the activities can be skipped, i.e., they have an exclusive gateway upfront. The lifecycles of the data objects are discovered and represented in the original process model, as depicted in Figure 7.

Each data output edge shows the number of instances that the activity is writing over the number of instances the activity occurs, represented in percentage. For example, the data objects discovered from the case attributes are written in $100 \%$ of the times when the respective activity (New) is executed.

\footnotetext{
${ }^{1}$ https://github.com/DorinaBano/dataObjectDiscovery.git
} 


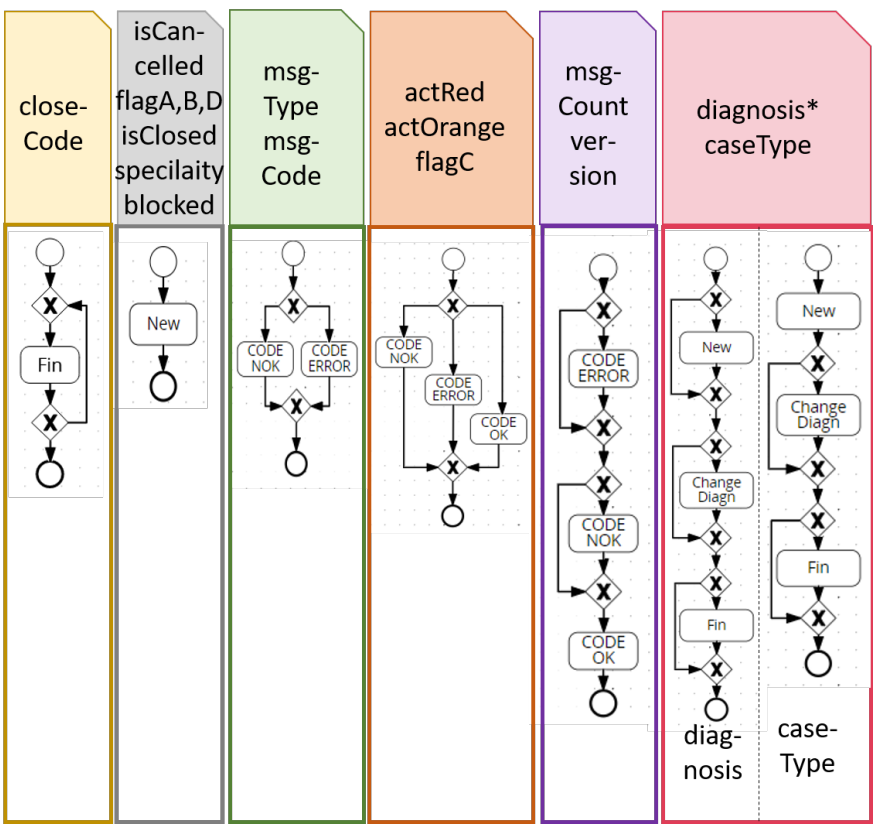

Figure 6: Discovered data objects and their attribute-access behavior for the Hospital Billing event log (color figure is available online).

We argue that enriching the process model with data objects and their lifecycles helps the process experts to better understand the process execution. For example, the Hospital Billing process model illustrated in Figure 7 contains a parallel gateway. Focusing on the data object that contains the msgCount and version attribute, it can be observed that the Code Error and the Code Ok activities access the data object in parallel to the Code Nok activity. However, judging from the data object lifecycle (see Figure 7) these activities should all be in a sequential relation. In this specific case, we investigate this discrepancy between the process model, the data object lifecycle and the original event log. The following is observed:

- there are only 8 cases out of 100.000 in the event log where the Code $\mathrm{Ok}$ activity is eventually followed by the Code Error activity. This is due to the presence of a loop in the discovered process model (not shown in Figure 7 due to the low frequency), where the Code Error activity happens a second time. However, the second time the Code Error activity does not access the data object again. Hence, the data object lifecycle manifests only a sequential state change.

- there are 13 cases where the Code Ok activity is eventually followed by the Code Nok activity, but in none of these cases the data object is overwritten by the latter.

The RTFM event log involves events regarding the road traffic fines issued by the Italian police. It includes 150.370 cases (561.470 events) that are processed by the municipality over thirteen years. The event log contains 11 activities and only 7 of them are accessing (writing or modifying) an event $\log$ attribute. The discovered process model from the original

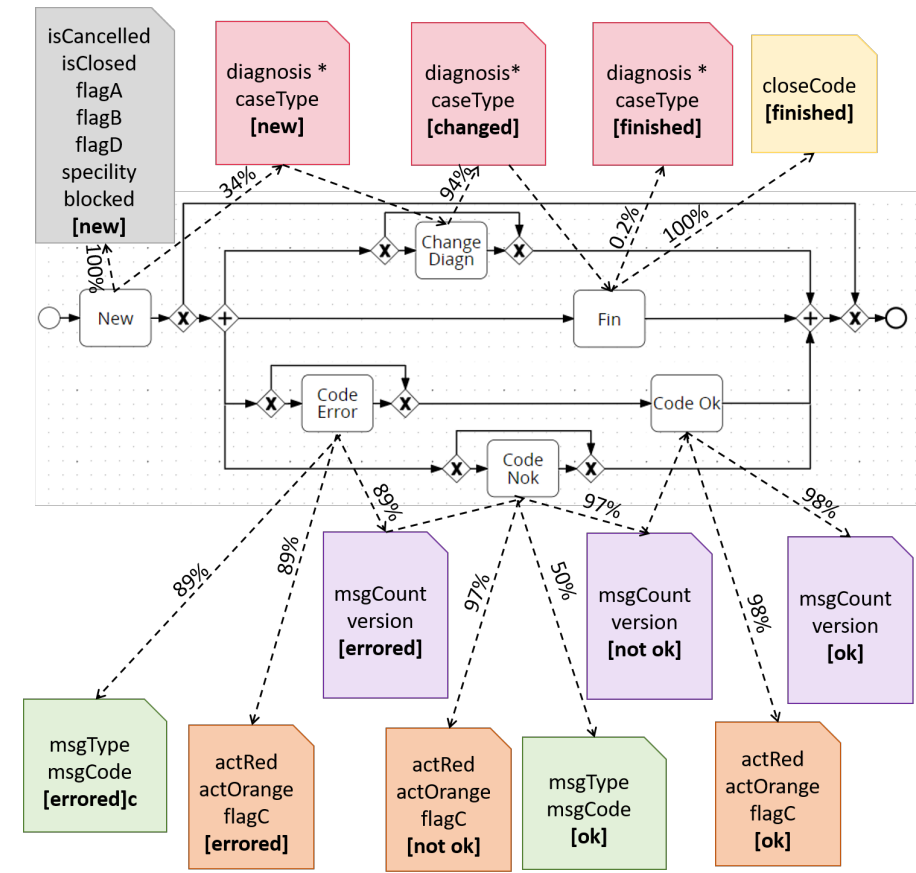

Figure 7: Process model (discovered by the Inductive Visual Miner), data objects and their lifecycles for the Hospital Billing event log (color figure is available online).

event $\log$ (the activities that do not access any attribute are removed) together with the data objects and their lifecycles are illustrated in Figure 8.

Compared to the Hospital Billing event log, the RTFM $\log$ contains more static data objects (i.e., their states do not change during process execution). That is why there are no activities that read something from the data objects and later change their state. In addition, 2 out of 8 discovered data objects are created as a result of case attributes. Another difference compared to the Hospital billing event log is that there are more activities that manifest a $100 \%$ writing ratio.

Discussion. Enriching the discovered process model with data objects helps the process experts to understand how data is manipulated through business process execution. The discrepancies between the process model and the data objects lifecycles together with the access rates can shed light on how the data-flow matches the control-flow. This is due to the fact that the relation between the business process activities and data objects are clearly defined.

\section{RELATED WORK}

Fahland et al. [28] focuses on artifact-centric process mining which is an extension of process mining and can analyze the event logs with more than one case identifier. The approach takes as input an event log or a relational database and outputs a data model of objects together with their relations and a process model called artifact-centric process model. The process model describes the behavior of each data object and the dependencies between them. The approach assumes the association of each event to one data object and from the 


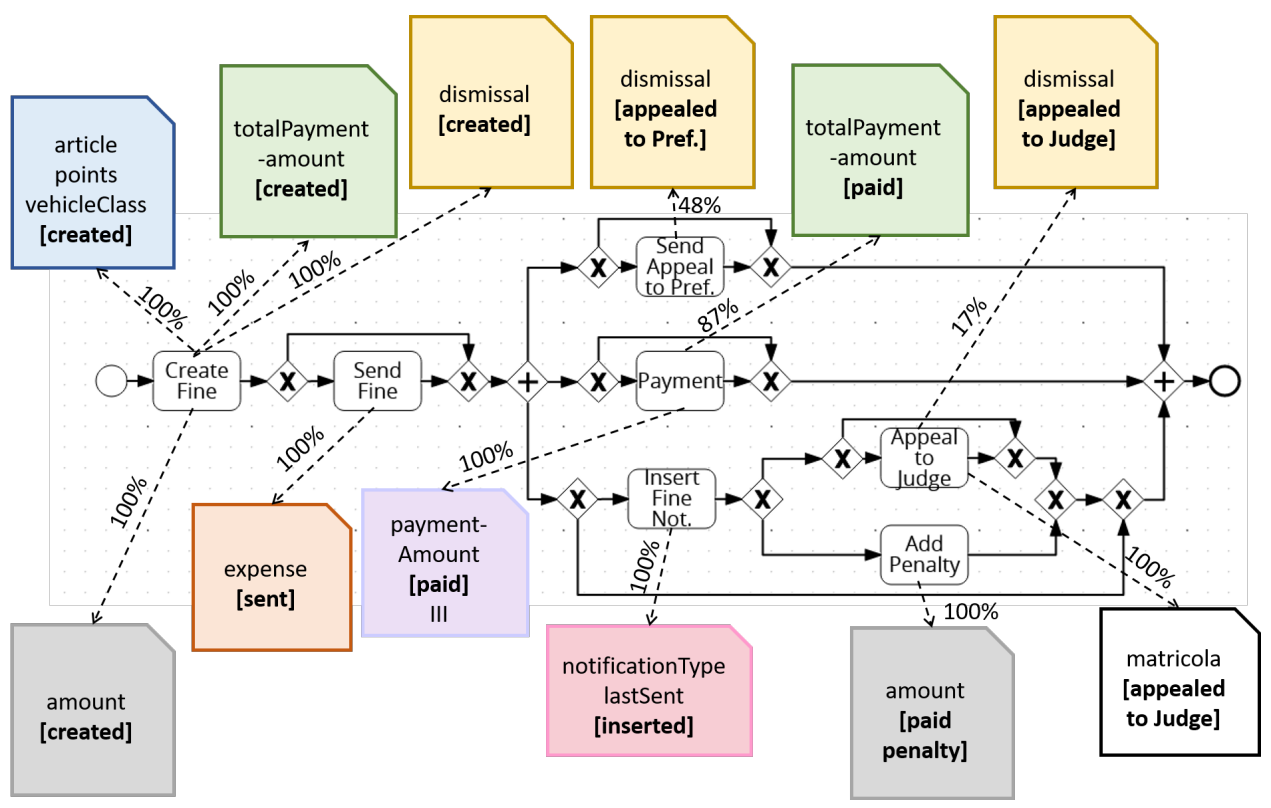

Figure 8: Process model (discovered by the Inductive Visual Miner), data objects and their lifecycles for the Road Traffic Fine Management event $\log$ (color figure is available online).

behavior relations between all events pertaining to one data object, the artifact lifecycle is discovered. Afterwards, the behavior dependencies between different artifacts are defined. In this way, the complete artifact-centric process model provides not only the lifecycle model of each artifact but also the dependencies and their cardinalities between different artifacts. Different from our approach, which requires as input an event $\log$, artifact-centric process discovery requires an event $\log$ with multiple case identifiers (multiple case notion event log). In addition, the data model is required as input and assumes that each event is assigned to one data object. This is a strong assumption because event logs, in the classical process mining sense, are unaware of data objects. They, rather, contain information about certain attributes. It is the focus of this paper to categorize event log attributes into data objects, not just based on static information (see [29]), but on the behavior aspects of these attributes.

The previously discussed work has led to a new process mining paradigm, object-centric process mining [30]. The input event log with multiple case notions called eXtensible Object-Centric (XOC) contains explicit information about the relation between events and objects. In eXtensible Event Stream (XES) logs [1], which are used in this paper, this information is not explicitly captured. The output of objectcentric process mining is the behavior of all objects and their relations (hence the name) while the output of classical process mining is a process model where the control-flow is the focus and data-flow is treated as a second-class citizen. Our paper indeed captures this implicit information, which we show helps to improve the overall understanding of the process execution.

Leoni et al. in [7] and [31] aim to analyze, via the data-flow perspective, why some cases take a certain process execution path. Using any traditional process mining discovery technique a process model is discovered. Then, the alignment between the event $\log$ and discovered process model is computed. By looking at each decision point as a classification model, machine learning techniques are used to discover guards. In the same vein, Mannhardt et al. [32] propose an approach to check process conformance in a balanced way, taking into account different process perspectives (i.e., control-flow, data, resources, and time perspectives). In contrast to the approach presented in [31] in which the control-flow is the most important perspective for identifying deviation, the authors emphasize that balancing the perspective in a customizable manner delivers more meaningful results. To depict one type of explanation for deviations over the other the cost function is defined. The output of these approaches is a Petri net with data. Different from our approach, the authors consider the attribute values to generate rules on how attributes affect the controlflow. In our approach, we focus on how the control-flow affects the collective behavior of attributes (data objects behavior). Similar to our approach, the number of event log attributes plays an important role in the accuracy of the findings.

Mannhardt et al. in [16] propose an approach that discovers the process model while considering the event labels and data attributes. In addition, classification techniques are used to discover data dependencies between the process model activities. The process model is discovered based on the Heuristic Miner algorithm and is enriched with the data dependencies. Similar to our approach the discovered process model reveals information about the control- and data-flows but this information is discovered and presented to the end user in a different way. In contrast to our approach the authors are more focused on the actual attribute values of an event log 
while we rely on the relation between activities and attributes names regarding the write or update access of their values.

\section{CONCLUSION}

In this paper, we present an approach to discover the data objects and their lifecycles from an event log that is tailored for process mining. To achieve this, we analyze how the event log attribute values change for each case and group these attributes into data objects, which reflect how the business process activities consume the data during the process execution. The process model is enhanced with data object lifecycles and execution heuristics stemming from the event log.

We evaluate our approach with two real-life event logs. Our evaluation shows that the discovered process model can reveal more behavioral information to the process expert when it is enhanced with data objects and their lifecycles. Thus, the enhanced process model is closer to the real-world execution of the process in terms of semantics.

In future work, we plan to introduce our solution to a few process experts to observe and measure how they apply the presented method. In addition, we would like to look at a set of event logs extracted from the same database but serving different business goals in order to analyze data objects manipulation across different business processes.

\section{REFERENCES}

[1] C. Gunther and H. Verbeek, XES - standard definition, ser. BPM reports. BPMcenter.org, 2014, vol. 1409.

[2] W. M. van der Aalst, "A practitioner's guide to process mining: Limitations of the directly-follows graph," Procedia Computer Science, vol. 164, pp. 321-328, 2019.

[3] W. van der Aalst, Process Mining-Data Science in Action, 2nd ed. Berlin Heidelberg: Springer, 2016.

[4] A. Weijters, W. Aalst, and A. Medeiros, "Process Mining with the Heuristics Miner-algorithm," BETA Working Paper Series, WP 166, Eindhoven University of Technology, Eindhoven, 2006.

[5] S. J. J. Leemans, D. Fahland, and W. M. P. van der Aalst, "Process and deviation exploration with inductive visual miner," in Business Process Management Demos BPM Demos, ser. CEUR Workshop Proceedings, vol. 1295. CEUR-WS.org, 2014, p. 46.

[6] C. W. Günther and W. M. Van Der Aalst, "Fuzzy mining-adaptive process simplification based on multi-perspective metrics," in International conference on business process management. Springer, 2007, pp. 328343.

[7] M. de Leoni and W. M. P. van der Aalst, "Data-aware process mining: Discovering decisions in processes using alignments," in Proceedings of the 28th Annual ACM Symposium on Applied Computing, ser. SAC '13. New York, NY, USA: Association for Computing Machinery, 2013, p. 1454-1461.

[8] M. Reichert, "Process and data: Two sides of the same coin?" in On the Move to Meaningful Internet Systems: OTM 2012, Confederated International Conferences: CoopIS, DOA-SVI, and ODBASE 2012, Rome, Italy, September 10-14, 2012. Proceedings, Part I, ser. Lecture Notes in Computer Science, vol. 7565 . Springer, 2012, pp. 2-19.

[9] K. Diba, K. Batoulis, M. Weidlich, and M. Weske, "Extraction, correlation, and abstraction of event data for process mining," Wiley Interdisciplinary Reviews: Data Mining and Knowledge Discovery, p. e1346, 2019.

[10] C. Combi, B. Oliboni, M. Weske, and F. Zerbato, "Conceptual modeling of processes and data: Connecting different perspectives," in International Conference on Conceptual Modeling (ER), ser. LNCS, vol. 11157. Springer, 2018, pp. 236-250.

[11] A. Meyer, L. Pufahl, D. Fahland, and M. Weske, "Modeling and enacting complex data dependencies in business processes," in 11th Int. Conf. Business Process Management (BPM), ser. LNCS, F. Daniel, J. Wang, and B. Weber, Eds., vol. 8094. Springer, 2013, pp. 171-186.
[12] N. Trčka, W. M. P. van der Aalst, and N. Sidorova, "Data-flow antipatterns: Discovering data-flow errors in workflows," in Advanced Information Systems Engineering, P. van Eck, J. Gordijn, and R. Wieringa, Eds. Berlin, Heidelberg: Springer Berlin Heidelberg, 2009, pp. 425439

[13] D. Calvanese, S. Ghilardi, A. Gianola, M. Montali, and A. Rivkin, "Formal modeling and smt-based parameterized verification of data-aware bpmn," in International Conference on Business Process Management. Springer, 2019, pp. 157-175.

[14] C. Combi, B. Oliboni, M. Weske, and F. Zerbato, "Conceptual modeling of inter-dependencies between processes and data," in Proceedings of the 33rd Annual ACM Symposium on Applied Computing, ser. SAC '18. New York, NY, USA: ACM, 2018, p. 110-119.

[15] S. Schönig, C. Di Ciccio, F. M. Maggi, and J. Mendling, "Discovery of multi-perspective declarative process models," in Service-Oriented Computing, Q. Z. Sheng, E. Stroulia, S. Tata, and S. Bhiri, Eds. Cham: Springer International Publishing, 2016, pp. 87-103.

[16] F. Mannhardt, M. de Leoni, H. A. Reijers, and W. M. P. van der Aalst, "Data-driven process discovery - revealing conditional infrequent behavior from event logs," in Advanced Information Systems Engineering, E. Dubois and K. Pohl, Eds. Cham: Springer International Publishing, 2017 , pp. $545-560$.

[17] Object Management Group, "Business Process Model and Notation (BPMN), v2.0.2," http://www.omg.org/spec/BPMN/2.0.2/.

[18] M. Weske, Business Process Management: Concepts, Languages, Architectures, 3rd ed. Berlin Heidelberg: Springer-Verlag, 2019.

[19] E. Gonzalez Lopez de Murillas, "Process mining on databases: extracting event data from real-life data sources," Ph.D. dissertation, Mathematics and Computer Science, Feb. 2019, proefschrift.

[20] R. J. C. Bose, R. S. Mans, and W. M. van der Aalst, "Wanna improve process mining results?" in 2013 IEEE symposium on computational intelligence and data mining (CIDM). IEEE, 2013, pp. 127-134.

[21] B. van Dongen, R. Dijkman, and J. Mendling, "Measuring similarity between business process models," in Advanced Information Systems Engineering, Z. Bellahsène and M. Léonard, Eds. Berlin, Heidelberg: Springer Berlin Heidelberg, 2008, pp. 450-464.

[22] R. Uba, M. Dumas, L. García-Bañuelos, and M. La Rosa, "Clone detection in repositories of business process models," in Business Process Management, S. Rinderle-Ma, F. Toumani, and K. Wolf, Eds. Berlin, Heidelberg: Springer Berlin Heidelberg, 2011, pp. 248-264.

[23] H. Leopold, S. Smirnov, and J. Mendling, "On the refactoring of activity labels in business process models," Inf. Syst., vol. 37, no. 5, pp. 443-459, 2012. [Online]. Available: https://doi.org/10.1016/j.is.2012.01.004

[24] J. Mendling, H. A. Reijers, and J. Recker, "Activity labeling in process modeling: Empirical insights and recommendations," Information Systems, vol. 35, no. 4, pp. 467-482, 2010.

[25] Mannhardt, Felix, "Hospital Billing - Event Log," 2017, 4TU.ResearchData. Dataset. https://doi.org/10.4121/uuid:76c46b83c930-4798-a1c9-4be94dfeb741.

[26] de Leoni, M. (Massimiliano); Mannhardt, Felix, "Road traffic fine management process," 2015, 4TU.ResearchData. Dataset. https://doi.org/10.4121/uuid:270fd440-1057-4fb9-89a9-b699b47990f5.

[27] A. Berti, S. J. van Zelst, and W. van der Aalst, "Process mining for python (PM4Py): Bridging the gap between process- and data science," in Proceedings of the ICPM Demo Track 2019, 2019, pp. 13-16. [Online]. Available: http://ceur-ws.org/Vol-2374/

[28] D. Fahland, "Artifact-centric process mining," in Encyclopedia of Big Data Technologies, S. Sakr and A. Y. Zomaya, Eds. Springer, 2019.

[29] D. Bano and M. Weske, "Discovering data models from event logs," in Conceptual Modeling, G. Dobbie, U. Frank, G. Kappel, S. W. Liddle, and H. C. Mayr, Eds. Cham: Springer International Publishing, 2020, pp. $62-76$

[30] W. M. P. van der Aalst, "Object-centric process mining: Dealing with divergence and convergence in event data," in Software Engineering and Formal Methods, P. C. Ölveczky and G. Salaün, Eds. Cham: Springer International Publishing, 2019, pp. 3-25.

[31] M. de Leoni and W. M. P. van der Aalst, "Aligning event logs and process models for multi-perspective conformance checking: An approach based on integer linear programming," in Business Process Management, F. Daniel, J. Wang, and B. Weber, Eds. Berlin, Heidelberg: Springer Berlin Heidelberg, 2013, pp. 113-129.

[32] F. Mannhardt, M. De Leoni, H. A. Reijers, and W. M. Van Der Aalst, "Balanced multi-perspective checking of process conformance," Computing, vol. 98, no. 4, pp. 407-437, 2016. 